\title{
Biotopologie et dimorphisme \\ du Sporotrichum schenckii (Hetkoen et Perkins 1900)
}

\author{
par Monique THIBAUT
}

Laboratoire de Parasitologie et de Mycologie ( $\mathrm{P}^{\mathrm{r}}$ M. LARIVIÈRE), U.E.R. des Cordeliers 15, rue de l'Ecole-de-Médecine, F 75 - Paris-6

\begin{abstract}
Résumé
Sporotrichum schenckii (Hetkoen et Perkins, 1900) peut mener une vie saprophytique indépendante dans l'environnement humain. Ce champignon pathogène a été isolé de la végétation et du sol, mais les autres agents de dissémination sont l'air, l'eau, les insectes et les animaux. Une certaine humidité relative est nécessaire à la croissance du champignon. La sporotrichose est une maladie des sujets manipulant la terre, et, surtout, les végétaux. Certains caractères épidémiologiques du Sporotrichum schenckii sont rapportés, en accentuant le rôle des facteurs favorables à la croissance du champignon.
\end{abstract}

\section{Summary}

Sporotrichum schenckii (Hetkoen \& Perkins, 1900) may have an independant saprophytic existence in man's environment. This pathogenic fungus has been isolated from vegetation and soil, but the other agents of dissemination are air, water, insects and animals. Varying relative humidities influence the growth of the fungus. Sporotrichosis also occurs in persons exposed to soil and above all to plants. Certain epidemiological characteristics of Sporotrichum schenckii are reported with emphasis on factors for growth of this fungus. 
Depuis la fin du siècle dernier, de nombreux travaux se sont attachés à l'étude de la biologie du Sporotrichum schenckii (Hetkoen et Perkins 1900). Certains d'entre eux ont précisé l'écologie du champignon dans sa forme saprophytique. D'autres ont étudié le parasitisme, facultatif, de ce Sporotrichum chez l'animal et l'homme. Ces recherches sont très diverses; il nous a paru nécessaire de les grouper dans un travail d'ensemble, afin d'examiner les différents problèmes qu'elles ont posé.

\section{I. - BIOTOPOLOGIE}

La connaissance des lieux où vit le Sporotrichum schenckii présente une importance capitale pour comprendre comment s'infestent l'homme et les animaux. L'agent de la sporotrichose vit en saprophyte dans la nature, dans le sol sur des fragments de végétaux ou des détritus divers. L'homme ou l'animal s'inoculent le champignon, le plus souvent par l'intermédiaire de débris végétaux ou d'objets souillés par ces débris. En effet, ce Sporotrichum végète partout, même sur des milieux pauvres, tels que les écorces et les épines (de Beurmann et Gougerot 1907), aussi la maladie prédomine-t-elle dans les zones rurales, où les travailleurs sont fréquemment exposés par leur profession à des traumatismes relevant de ces conditions. L'infection suit habituellement l'introduction traumatique dans le derme du Sporotrichum schenckii. L'existence dans la nature, à l'état saprophytique, de cet Adélomycète, potentiellement pathogène, constitue un fait d'un grand intérêt épidémiologique. Nous allons passer en revue les différents facteurs jouant un rôle dans sa biologie.

\section{A. L'air.}

Il intervient par les xérospores qui peuvent s'y trouver et par son humidité, favorisant leur culture.

\section{1) Xérospores}

Les conidies du Sporotrichum schenckii étant des xérospores sont entraînées par les courants d'air et le vent, qui les détachent des hyphes. Simson (1947) exposa à l'air des boîtes de Pétri, contenant un milieu à base d'agar et à partir d'une de ces boîtes, isola un Sporotrichum identique en tous points à l'espèce pathogène. De nombreuses expériences ont été effectuées dans les mines du Witwatersrand, en Afrique du Sud, pour vérifier que les spores sont bien' disséminées par les courants d'air (Brown, Weintroub et Simson 1947).

\section{2) Température extérieure et humidité de l'air}

Les conditions climatiques jouent un rôle important dans le développement de la sporotrichose et expliquent les zones d'endémicité. Mackinnon (1948) a montré qu'il y a une distribution saisonnière des cas de sporotrichose en Uruguay: la mycose s'observe plus fréquemment entre mars et juillet, lorsque la température et l'humi- 
dité sont plus favorables à la croissance du Sporotrichum schenckii. Mais le champignon peut se développer entre les températures extrêmes de $10^{\circ}$ et $38^{\circ} \mathrm{C}$ (Mackinnon 1968). L'optimum de température, surtout sur les milieux humides, se trouve entre $20^{\circ}$ et $30^{\circ}$. Les spores résistent à $0^{\circ}$ et à $+55^{\circ}$. Aux températures ordinaires, elles restent vivantes pendant des années. Elles sont peu sensibles à l'action des rayons solaires et des intempéries: des cultures laissées à l'air libre sont encore vivantes un an après (de Beurmann et Gougerot 1907). La résistance de ce Sporotrichum est donc très grande.

Le degré hygrométrique de l'air, intervient en favorisant la culture sur différents substrats (Mackinnon 1948). La température et l'humidité de l'air sont des facteurs jouant un rôle, sur la croissance active de ce micro-organisme sur le bois et sont aussi favorables au développement d'autres champignons.

Le Sporotrichum schenckii crôt sur le bois, à une humidité relative allant de $100 \%$ à $92 \%$ (Brown, Weintroub et Simson 1947). Le nombre des spores libérées est influencé par l'humidité contenue dans le bois.

\section{B. Le sol.}

Une importante source de contamination par le Sporotrichum schenckii, est représentée par le sol, où le champignon vit en saprophyte et dont il a été isolé par Emmons (1951). Le terme de sol est employé au sens large: c'est un mélange complexe de débris organiques tels que matériel végétal corrompu, humus, excreta, restes kératinophiles et protéiques d'insectes, oiseaux et animaux divers, ainsi que la microflore et la microfaune vastes et variées du sol. Ce Sporotrichum crô̂t dans tous les types de sol. Il y trouve les différents éléments nutritifs nécessaires à son développement saprophytique, en particulier la pyrimidine, qui est un bon facteur de croissance, pour les souches potentiellement pathogènes (Drouhet et Mariat 1952, Howard et Orr 1963). Emmons (1951) a cultivé le Sporotrichum schenckii à partir du sol et a montré qu'il s'y trouvait beaucoup moins abondant que d'autres champignons pathogènes, tels que le Cryptococcus neoformans et l'Histoplasma capsulatum. Cet auteur a trouvé deux fois seulement le Sporotrichum schenckii, sur 175 spécimens de sols examinés. Plus récemment, au Brésil, Rogers et Beneke (1964) ont cultivé ce champignon à partir du sol. Mais il faut savoir distinguer l'espèce schenckii, d'autres espèces, qui se trouvent également dans le sol. La terre peut être à l'origine de la sporotrichose, par exemple, chez des fabricants de briques crues ou des joueurs de football (Lavalle 1949). La boue permet la croissance saprophytique du champignon (Du Toit 1942, Brown, Weintroub et Simson 1947).

\section{L'eau.}

L'eau peut également être contaminée par des spores et devenir ainsi une source d'infection. Du Toit (1942) a montré que le Sporotrichum schenckii pouvait y pousser en vie saprophytique. L'eau est, d'autre part, un agent actif dans la séparation 
et la dispersion des conidies. Si une goutte d'eau vient en contact avec une culture, les spores sont libérées immédiatement dans le liquide. Leur dispersion consécutive dépend de la direction de l'écoulement de l'eau (Brown, Weintroub et Simson 1947). Mackinnon (1948) a observé que, dans la nature, une bonne croissance du champignon était soumise à un haut degré d'humidité relative, une température supérieure à $15^{\circ}$ et des chutes de pluie répétées, ces différentes conditions devant persister pendant un minimum de 3 à 4 jours.

\section{Les végétaux.}

Différentes plantes ont été incriminées, comme source d'infestation: des phanérogames ou des cryptogames; des arbres, des buissons ou des plantes herbacées; la végétation morte ou vivante. Le Sporotrichum schenckii vit, en effet, en saprophyte, sur des plantes mortes ou malades, qui constituent un milieu favorable à sa multiplication et sont ainsi à l'origine de la presque totalité des cas de sporotrichose. Sur les bois morts, où il peut croître, il forme des plaques tantôt mates, brunes ou noirâtres, tantôt visqueuses. La dessiccation peut leur donner un aspect fuligineux. Les éléments ligneux sur lesquels pousse le champignon peuvent devenir infestants. La maladie est fréquente chez les maîtresses de maison, qui utilisent des fibres végétales pour les travaux domestiques, ainsi que chez les charpentiers, bûcherons, fleuristes, agriculteurs et autres travailleurs employant du matériel végétal. Les conidies du Sporotrichum schenckii peuvent en effet vivre très longtemps sur la végétation, lorsque les conditions sont favorables (Brown, Weintroub et Simson 1947).

\section{1) Parmi des Cryptogames}

Les mousses et en particulier les sphaignes, manipulées par les fleuristes, renferment souvent le Sporotrichum schenckii (Emmons 1951). D'Alessio, Leavens, Strumpf et Smith (1965) ont relaté une épidémie de sporotrichose dans le Vermont, où la source d'infestation était constituée par les sphaignes. Les sujets atteints étaient tous des pépiniéristes, en contact avec cette Muscinée, à partir de laquelle, on isola le Sporotrichum schenckii.

\section{2) Parmi les Phanérogames}

Les fragments de tiges pourries, d'œillets, rosiers, glaïeuls, épine vinette et d'autres fleurs, constituent un milieu favorable au développement de ce Sporotrichum. Les piqûres d'épines ou de plantes épineuses, les échardes, inoculent le champignon. Emmons a rapporté six cas de sporotrichose, observés chez des fleuristes. Foerster (1926) a relaté quatorze cas de cette maladie, survenus parmi les ouvriers d'une pépinière, qui avaient été infectés, de manière indiscutable, par des piqûres d'épine vinette. Le Sporotrichum schenckii a été isolé des épines de Berberis thunbergii et Berberis vulgaris. Le cactus Cereus a été à l'origine de cas de sporotrichose (Londero 
et Cercal 1956). Mariat (1968) a observé le cas d'une laborantine, contaminée par un Aechmea. Le champignon fut isolé des feuilles épineuses de cette Broméliaceae décorative, ainsi que de la terre sur laquelle poussait cette plante. La plante contaminée fut repiquée sur un sol vierge. Deux mois plus tard, la totalité du nouveau sol était infesté.

La présence du Sporotrichum schenckii, vivant en saprophyte sur des végétaux, a été signalée, pour la première fois, par de Beurmann et Gougerot (1907). Sur les légumes vendus aux halles, par leur malade $n^{\circ}$ XII, ils ont mis en évidence le champignon. Ces auteurs ont découvert le Sporotrichum beurmanni sur l'écorce d'un hêtre, sur des prêles et sur des graines d'Avena sativa (1908). Sartory (1915) en a isolé une souche, sur des grains de blé.

Le champignon a également une prédilection pour les foins, dans les prés salés. Des épidémies familiales ou professionnelles ont été observées au Mexique, chez les potiers et les personnes manipulant des graminées servant à l'emballage des poteries (Gonzales Benavides 1952). Entassées en milieu humide, elles servent de milieu de culture au Sporotrichum et renferment des spores infectantes, qui sont inoculées par les échardes de bois et les clous, lors de la manutention (Ansel 1963). A partir de ces emballages végétaux, Garrett et Robbins (1960) ont obtenu des cultures du Sporotrichum schenckii. Bazex et al. (1961) ont décrit un cas de sporotrichose survenu à la suite d'une piqûre avec un fil de fer, entourant des balles de vieux papiers. Plus récemment (1968), Balabanoff a rapporté une épidémie de sporotrichose, survenue parmi les ouvriers d'une papeterie. Ces ouvriers transportaient des cartonnages venant d'un atelier très humide, dans un bâtiment de bois où les rats fourmillaient. Le carton était fabriqué à partir de certains conifères, qui sont un réservoir naturel du Sporotrichum. Du Toit (1942) a montré la présence de cet Adélomycète, sur les bois de charpente. Le bois semble un milieu très adapté pour sa croissance saprophytique. Le Sporotrichum schenckii prolifère, en effet, particulièrement, sur les bois de charpente des mines qui, grâce à l'obscurité, à la chaleur et à l'humidité, offrent des conditions favorables à la vie saprophytique. Ainsi s'expliquent les épidémies considérables de sporotrichose, survenues dans les mines d'or du Witwatersrand, en Afrique du Sud (Brown, Weintroub et Simson 1947). Ces auteurs ont réussi à cultiver le Sporotrichum sur différents échantillons de bois, maintenus à une humidité relative très élevée. Certains bois, en effet, tel celui de l'Acacia mollissima, constituent un substrat très favorable à sa culture. La pénétration du champignon dans le bois est, en général, inférieure à $1 / 2$ millimètre, la croissance étant confinée, aux couches les plus externes, où a lieu une sporulation profuse. Cependant, le Sporotrichum peut pénétrer le bois beaucoup plus profondément, les hyphes et les conidies étant parfois trouvées à deux centimètres de la surface. Cela est probablement dû au fait que des spores détachées ont pu être aspirées dans les fibres ou les vaisseaux du bois par un phénomène de capillarité et ont alors germé. D'autres bois, au contraire, tel celui du Pseudotsuga taxifolia, constituent un milieu absolument défavorable au développement du Sporotrichum schenckii. Pour les auteurs sud-africains, le bois a été, sans aucun doute, la principale source d'infection, au cours de l'épidé- 
mie du Witwatersrand. En effet, lorsqu'on traita les bois de charpente des mines, l'incidence de la sporotrichose décrut considérablement. Les auteurs sud-africains ont également montré que le développement de champignons Polyporacées ou Corticiacées empêche celui du Sporotrichum schenckii.

\section{E. Les animaux.}

\section{1) Sporotrichose spontanée}

L'infection à Sporotrichum existe à l'état spontané chez différents animaux. En effet, les animaux, tout comme l'air, le sol, l'eau et les végétaux, peuvent être considérés comme des porteurs de germes. Ils peuvent l'être passivement ou activement (Ainsworth et Austwick 1959). La contamination directe des humains par des animaux sporotrichosiques n'a été qu'assez rarement observée. Plus souvent, les animaux infestés jouent un rôle de réservoir.

a) Oiseaux :

Rouslacroix a isolé le champignon, chez le perroquet, la poule et différents oiseaux. Les coups de bec de poules et d'autres oiseaux peuvent donc transmettre la maladie (1912).

b) Rongeurs :

La sporotrichose spontanée a été observée chez le rat gris et le rat blanc par Lutz et Splendore (1908). Ces auteurs, en examinant systématiquement des rats, lors d'une épidémie de peste à Sao Paulo, découvrirent un certain nombre de rongeurs atteints d'abcès chroniques. A partir du pus des abcès, ils cultivèrent un champignon filamenteux. Peu après, Lutz et Splendore retrouvèrent plusieurs cas spontanés de la même mycose sur des rats blancs élevés dans leur laboratoire. Ils réunirent ainsi une quarantaine de cas de la maladie, dont l'agent fut identifié au Sporotrichum beurmannii. L'étiologie de la mycose spontanée du rat doit être semblable à celle de l'homme: elle est probablement due à l'inoculation par les végétaux, mais elle peut aussi se transmettre d'un animal à l'autre par morsure. Lutz et Splendore ont également rapporté l'histoire d'un infirmier contaminé par la morsure d'un rat sauvage. Valle Meza et Barba Rubio (1961) ont relaté l'observation d'un bébé mordu par un rat à l'âge de 2 jours et qui, huit jours plus tard, développa une sporotrichose.

c) Herbivores :

Chaillot a signalé la maladie chez le chameau à Beyroüh (1932). Carougeau (1909) a découvert la sporotrichose spontanée du mulet et du cheval. Cette affection est très fréquente à Madagascar. Le Sporotrichum, vivant dans le monde extérieur, est inoculé par la peau ou les muqueuses. La contamination peut se faire à l'occasion de blessures dues au harnachement ou de plaies accidentelles. Mais souvent la porte d'entrée passe inaperçue. 
d) Carnivores :

Gougerot et Caraven (1908) ont observé trois chiens atteints de sporotrichose, due au Sporotrichum beurmannii. La maladie a même été observée chez un Boa constrictor.

e) INSECTES :

Le Sporotrichum schenckii se développe sur certains insectes vivants, en particulier sur les mouches, les guêpes, les fourmis. Les auteurs sud-africains ont démontré que les mouches (Musca domestica) peuvent s'infecter au contact d'ulcères de malades sporotrichosiques. L'observation leur a montré que le Sporotrichum schenckii pouvait être isolé des pattes de mouches et de leurs excreta. Ils ont également démontré que le champignon reste vivant, pendant plusieurs jours, dans l'intérieur de la mouche.

\section{2) Sporotrichose expérimentale}

Schenk (1898), par voie sous-cutanée, reproduisit chez le chien, l'un des principaux caractères de l'affection humaine, la gomme, et la rétroculture fut positive. Hetkoen (1900) obtint, chez deux souris blanches, une pyémie avec infection généralisée. La dissection montra des lésions dans le foie, la rate et les ganglions abdominaux. A partir de ces organes, il isola le Sporotrichum schenckii. De Beurmann et Ramond n'eurent que des insuccès. Puis de Beurmann et Gougerot (1912) reproduisirent expérimentalement la maladie par deux méthodes: inoculation sous-cutanée à des animaux nouveau-nés et inoculation à la patte du rat. Depuis lors, différents expérimentateurs ont obtenu la maladie chez un grand nombre d'animaux. Les animaux sensibles sont en particulier: le rat, la souris, le hamster, le cobaye, le lapin, le chien, le chat, le singe et même l'embryon de poulet dans l'œuf âgé de 12 jours, Les voies d'inoculation les plus utilisées sont l'intra-péritonéale et l'intra-testiculaire, ainsi que la racine de la patte en sous-cutanée.

\section{F. L'Homme.}

L'homme lui-même est une source d'infection. Il peut contaminer son entourage, ce qui peut expliquer de petites épidémies familiales, surtout entre enfants (Ansel 1963). La forme la plus commune est la sporotrichose dermato-lymphangitique, mais la maladie peut également atteindre les muqueuses et le poumon. Elle peut aussi se disséminer secondairement à la peau, aux os, au système nerveux et aux différents viscères.

\section{II. - DIMORPHISME DU SPOROTRICHUM SCHENCKII}

\section{A. Définition du dimorphisme.}

Le dimorphisme est la propriété fondamentale de certains champignons pathogènes, qui présentent une morphologie différente à $20^{\circ}$ : phase mycélienne, et à 
$37^{\circ}$ : phase levure, à condition que le milieu de culture utilisé à $37^{\circ}$ soit riche en protéines. La forme levure a une morphologie semblable à celle de la forme parasitaire du champignon.

Le Sporotrichum schenckii, champignon potentiellement pathogène de l'homme et des animaux, présente un intérêt particulier, car il montre réversiblement deux formes: la forme saprophytique filamenteuse et la forme parasitaire levuroïde. Il est possible d'obtenir in vitro la transformation de la forme filamenteuse en forme levure et le maintien de celle-ci, sous l'influence de facteurs divers. La morphologie de ce Sporotrichum est en effet différente à l'état parasitaire et à l'état saprophytique. En vie parasitaire, il se présente sous une forme levure. Cultivé au laboratoire sur milieu de Sabouraud ou sur milieu au moût de bière gélosé, il prend une forme filamenteuse, qui représente la phase mycélienne. On peut transformer la forme mycélienne en forme levure, en changeant la température du milieu et en modifiant sa composition.

\section{B. Saprophytisme et parasitisme.}

Le Sporotrichum schenckii, doué de pouvoir pathogène, ne vit pas normalement en parasite, mais bien en saprophyte. Comme on l'a vu plus haut, Beurmann et Gougerot, dans leur premier mémoire en 1906, ont en effet démontré son saprophytisme dans la nature. Ils ont obtenu des cultures de ce Sporotrichum sur tous les milieux naturels végétaux et animaux : paille, feuilles, grains, fruits, écorce, bois, épines, terre végétale, insectes morts, larves, chenilles et mouches vivantes. Ils ont également montré que les intermédiaires entre l'homme et le champignon peuvent être multiples : débris végétaux, insectes, animaux. Le Sporotrichum schenckii mène cette vie saprophytique dans le milieu extérieur: c'est un exosaprophyte. La voie de pénétration dans l'hôte, humain ou animal, peut varier.

Ce Sporotrichum est donc un parasite facultatif, pouvant passer de la vie saprophytique à la vie parasitaire. Mais, ce dernier mode de vie ne semble nullement nécessaire à son cycle évolutif et peut être considéré comme un simple phénomène d'adaptation accidentelle. Cette particularité biologique le distingue, en effet, des champignons parasites de plantes, qui sont souvent des parasites stricts, pouvant comporter un cycle évolutif obligatoire sur plusieurs espèces végétales.

La transformation immédiate d'un champignon pathogène, en sa forme saprophytıque, lorsqu'il passe de l'organisme animal ou humain, à un milieu de culture artificiel, est un phénomène très courant au laboratoire. Emmons (1951) a montré, expérimentalement, que les conidies qui se développent ensuite, sont plus résistantes aux influences néfastes de l'environnement, que les cellules très délicates de la phase parasitaire. De plus, les spores peuvent être produites en nombre considérable. II est facile de supposer alors, que les conidies sont les éléments les plus actifs, pour la dissémination du champignon dans la nature. Emmons a, d'autre part, observé expérimentalement, que les conidies sont infectantes pour l'animal, et que du fait de leur grande résistance, elles peuvent être des agents de contamination, beaucoup plus importants que la forme parasitaire. 


\section{La réduction morphologique parasitaire.}

Elle peut être considérée comme une manifestation du polymorphisme fungique. Cette réduction, qui est un phénomène très général en pathologie comparée, est particulièrement marquée chez les champignons parasites de l'homme et des animaux. Chez le Sporotrichum schenckii, elle se traduit par la présence d'éléments levure, parfois en navette (les corps en cigare), qui peuvent être intra ou extra-cellulaires chez l'hôte parasité.

\section{Facteurs permettant la transformation de la forme mycélienne en forme levure, in vitro.}

La phase levure ne s'obtient, in vitro, que très difficilement et en présence de conditions culturales complexes. De Beurmann et Gougerot (1912) avaient obtenu la forme levure de ce Sporotrichum, qu'ils nommaient «forme courte, oblongue, ovoïde », sur un milieu gélosé glucosé, recouvert d'une épaisse couche de pus. Davis (1913) avait obtenu les mêmes résultats, sur milieu au sang, recouvert d'huile de paraffine. Norden (1951) a eu des résultats comparables, sur des milieux à base d'organes animaux, contenant presque toujours du sang. Salvin (1949) a cultivé la forme levure du Sporotrichum schenckii sur un milieu à base de peptones. Bullen (1949) a utilisé avec succès un milieu peptoné, enrichi en gaz carbonique. Drouhet et Mariat (1952) ont mis au point un milieu liquide, riche en protéines, qui, agité à $37^{\circ}$, donne la forme levure du Sporotrichum, dans des conditions microaérophiles.

Les circonstances, qui font qu'un champignon pathogène dimorphique se présente plutôt sous la forme levure que sous la forme mycélienne, sont multiples. Ces différentes conditions sont réunies dans l'organisme parasité où se produit la transformation du mycélium en levures. Duncan a montré que cette transformation entrânait une augmentation de la virulence de l'agent pathogène.

On peut, in vitro, obtenir le passage de la forme saprophytique à la forme levure, grâce à l'influence des éléments suivants :

\section{1) Agents chimiques}

a) Protéines :

Le milieu de culture doit être enrichi avec de l'hydrolysat de caséine ou avec des acides aminés. L'arginine, l'alanine et le glycocolle sont les amino-acides qui donnent les meilleurs résultats. Ces acides, par leur décarboxylation, agiraient comme source d'anhydride carbonique. Pour Lwoff et Monod (1947), la composition de l'hydrolysat de caséine, très riche en acides aminés et en métabolites hétérocarboxyliques, expliquerait son excellent rendement en levures. 


\section{b) Oligo-Éléments :}

L'action catalytique de ceux de la solution de Berthelot est indispensable au dynamisme de la culture.

c) Facteurs vitaviniques:

D'après Oyama, cité par Robbins (1939), la croissance du Sporotrichum schenckii est améliorée par l'addition, à un mi.ieu synthétique, de polissures de riz ou d'aneurine (= thiamine). L'action favorisante de cette vitamine sur le champignon a été confirmée par Burkholder et Mayer (1943). La pyrimidine qu'elle contient est un facteur essentiel pour le développement des deux phases du Sporotrichum schenckii. La biotine est un facteur accessoire pour la croissance de l'une et l'autre forme (Drouhet et Mariat 1950).

d) AnHydride CARbonique :

L'atmosphère du milieu de culture doit être riche en gaz carbonique. Ceci est un facteur très important. On sait que l'hydrolysat de caséine et les acides aminés sont des sources d'anhydride carbonique.

Utter et Wood (1951) ont montré que la biotine agit comme fixateur de $\mathrm{CO}_{2}$. L'influence du gaz carbonique, entrevue par Bullen, a été mise en évidence par les travaux de Mariat et Drouhet (1952). Ces auteurs ont obtenu la forme levure du Sporotrichum schenckii, dans les tubes de milieu liquide où a barboté un mélange d'air et de $\mathrm{CO}_{2}$, quelle que soit la source d'azote. Une certaine tension d'anhydride carbonique suffit donc à provoquer la formation de la phase levure. Cependant, la forme levure peut être obtenue, sans apport de gaz carbonique, en utilisant un milieu liquide agité, contenant soit certains acides aminés (alanine, arginine ou glycocolle), soit un mélange d'acides aminés, soit de l'hydrolysat de caséine. Ces substances agiraient comme source de $\mathrm{CO}_{2}$ par leur décarboxylation. L'arginine du milieu de culture peut être transformée en acide $q$-cétoglutarique et en acide $a$-céto-aminoô-valérianique, composés capables de remplacer l'anhydride carbonique (Mariat 1952).

\section{2) Sang}

La présence de sang dans le milieu favorise la formation des levures, mais elle a aussi l'inconvénient de produire une filamentation de celles-ci. De toutes façons, le milieu au sang n'est pas obligatoire pour l'obtention de la forme levure.

\section{3) Agents physiques}

a) Température :

La transformation de la phase mycélienne en phase levure ne peut se faire qu'à la température de $37^{\circ}$. Entre $28^{\circ}$ et $37^{\circ}$, les formes levures filamentisent dans les cultures. A des températures inférieures à $28^{\circ}$, toutes les autres conditions étant identiques, c'est la phase mycélienne qui se développe (Mariat 1968).

b) $\mathrm{pH}$

Le $\mathrm{pH}$ optimum, pour le développement de la forme levure, en milieu liquide agité, est de 7,2. 


\section{4) Agents mécaniques : agitation}

Une agitation continue est nécessaire, pendant toute la durée de la culture, afin que le champignon puisse s'imprégner de tous les éléments constitutifs du milieu. D'autre part, l'accumulation d'anhydride carbonique, serait favorisée par l'agitation.

Pratiquement, on dispose les tubes de culture, en milieu liquide, sur un agitateur, animé de mouvements dans les trois dimensions de l'espace. L'agitateur, luimême placé dans une étuve à $37^{\circ}$, est mis en marche à raison de 100 secousses par minute.

\section{5) Durée de culture}

La forme levure du Sporotrichum schençkii est obtenue en quinze à vingt jours, à condition de respecter les facteurs précités.

\section{E. Mode de pénétration du champignon dans l'hôte animal ou humain, au cours de l'infection spontanée}

Le Sporotrichum schenckii provient du milieu extérieur, où il vit surtout en saprophyte sur des végétaux morts et dans le sol.

\section{1) Transmission par les animaux}

L'animal peut être un simple support du champignon ou au contraire un vecteur particulièrement actif du micro-organisme. L'infestation de l'animal se ferait à partir du sol.

La sporotrichose animale est transmissible à l'homme: Carougeau (1909) a cité le cas d'un vétérinaire, contaminé en se piquant au cours d'une intervention sur un mulet malade ; Hyde et Davis (1910) ont relaté l'observation d'un fermier sporotrichosique, habitant près d'une ferme où les chevaux étaient contaminés. Rouslacroix (1912) a observé un chancre sporotrichosiqụe du doigt, à la suite d'un coup de griffes de chien. La maladie survient fréquemment après une morsure par de petits mammifères (rats, furets, écureuils). Les insectes peuvent également véhiculer des conidies avec leurs pattes. La transmission du champignon par les mouches peut expliquer l'apparition de la sporotrichose faciale infantile.

\section{2) Transmission par les végétaux et le sol}

Le rôle des végétaux et du sol est beaucoup plus important que celui des animaux, dans l'infestation par le Sporotrichum schenckii.

a) PÉNÉtration pUlmonaire

Les spores se détachant des hyphes sont entraînées dans la poussière et dans l'air. Il peut $\mathrm{y}$ avoir inhalation de conidies en suspension dans l'atmosphère. Mais la 
rareté de l'infection respiratoire sporotrichosique, montre que ce mécanisme doit être exceptionnel.

\section{b) Pénétration digestive}

Elle peut se faire par l'ingestion de végétaux altérés, sur lesquels se trouve le Sporotrichum schenckii. Ce mode d'infestation est rare.

\section{c) Pénétration muqueuse}

Le champignon peut traverser les muqueuses des voies digestives supérieures. Les travaux de Thiry ont montré la présence du Sporotrichum schenckii dans la bouche de sujets sains. Par ailleurs, ce Sporotrichum peut également traverser la conjonctive. La conidie peut germer sur la conjonctive et la traverser, déterminant soit une lésion locale, soit un envahissement de tout l'organisme.

\section{Principales sources d'infestation par le Sporotrichum schenckii}

Dans l'ordre d'importance décroissante :

1. - Végétaux : écorces de hêtre, chêne, arbres abattus, prêles, épine-vinette, graminées, mousses (en particulier sphaignes), graines, fragments et débris végétaux.

2. - Sol.

3. - Piqûres d'insectes.

4. - Morsures d'animaux.

5. - Eau.

6. - Air.

Vecteurs du Sporotrichum schenckii :

- Epines ou aiguillons.

- Echardes.

- Fragments de paille.

- Insectes.

- Rongeurs.

- Chiens.

\section{d) PÉnétration cutanée}

Le Sporotrichum schenckii végète sur les épines et le bois, donc une piqûre, une écorchure peuvent l'introduire dans l'organisme. Il vit dans le sol, donc une plaie souillée de terre peut être le point de départ de la mycose. Le parasite n'attaque pas la peau saine. Il pén ̇̀tre dans l'organisme, soit par contamination de plaies préexistantes, soit par des frottements répétés au niveau d'une surface infectée de conidies, soit encore par des piqûres avec des échardes de bois ou des épines. Ce dernier mode de transmission est le plus fréquent. La sporotrichose s'observe donc surtout chez les 
jardiniers, les fleuristes, les agriculteurs, les bûcherons, les charpentiers, les employés des halles, les cuisiniers, qui se contaminent par piqûres de végétaux infestés. Le rôle du traumatisme est indéniable dans les premières observations de lymphangite sporotrichosique, publiées en Amérique: blessure du doigt avec un clou, chez le malade de Schenck, piqûre du doigt avec un fil de fer chez le malade de Brayton, blessure du doigt par un coup de marteau, chez le malade de Hetkoen et Perkins. Il semble bien que dans ces cas et un certain nombre d'autres, l'inoculation ait été directe, par un agent traumatisant contaminé.

L'exposé des différents travaux concernant la biologie du Sporotrichum schenckii nous a montré qu'elle présente un grand intérêt, d'une part à cause du dimorphisme de ce champignon, d'autre part à cause du rôle important joué par la végétation dans la transmission de la maladie.

\section{Professions eXPoséEs a L'INFestation PAR LE Sporotrichum schenckii}

- Professions dans lesquelles les sujets manipulent les végétaux et la terre: mineurs, bûcherons, charpentiers, jardiniers, fleuristes, agriculteurs, ménagères, potiers manipulant les pailles d'emballage (graminées), fabricants de briques (mélange de terre et d'herbes), équipes de football (au Mexique). fermiers.

- Professions dans lesquelles les sujets sont en contact avec des animaux : vétérinaires,

\section{Bibliographie}

Ainsworth (G.C.) et Austwick (P. K. C.), 1959. - In Fungal diseases of animals, Farnham Royal, Bucks: Commonwealth agricultural bureaux.

ANSEL (M.), 1957. - Mycoses et champignons parasites de l'homme, 1 vol., 346 p., Doin éd., Paris.

ANSEl D'IMEuX (M.), 1963. - Sporotrichose. Encyclopédie médico-chirurgicale, Maladies infectieuses, 8124, A 10, 1-4.

Balabanoff (V. A.), Koev (A.) et Stoynovski (V.), 1968. - Sporotrichose bei Arbeitern in einer Papierfabrik. Berufsdermatosen, 16, 261-70.

Bazex (A.), Dupré (A.), Parant (M.) et Bessière (L.), 1961. - Sporotrichose. Bull. Soc. fr. Derm. syph., 68 (3), 439.

Benham (R.W.) et Kesten (B.), 1932. - Sporotrichosis: its transmission to plants and animals, J. infect. Dis., 50, 437.

Brown (R.), Weintroub (D.) et Simson (M. W.), 1947. - Sporotrichosis infection in mines of the Witwatersrand. A symposium Transvaal chanber of Mines, éd. Johannesburg.

Brumpt (E.), 1949. - Précis de Parasitologie, 2 vol., Masson éd., Paris, 2.138 p.

Bullen (J. J.), 1949. - The yeast-like form of Cryptococcus farciminosus (Rivolta) : (Histoplasma farciminosum). J. Path. Bact., 61, 117-120. 
Campbell (C. C.), 1945. - Use of Francis'glucose blood agar in the isolation and cultivation of Sporotrichum schenckii. J. of Bact., 50, 235.

Carougeau, 1909. - Une nouvelle mycose des équidés. Journ. Méd. vétér., p. 8.

—, 1909. - Premier cas africain de sporotrichose de de Beurmann. Transmission de la sporotrichose du mulet à l'homme. Bull. et Mém. Soc. Méd. Hop. Paris, 34, 507.

Challlot. 1932. - Sporotrichose chronique chez un chameau. Rev. vét. milit.

Colles (P.), 1934. - Sporotrichose gommeuse disséminée chez un jeune chien. Bull. soc. sc. vét. Lyon, 37, 72-82.

D'Alessio (D.-J.), Leavens (L.-J.), Strumpf (G. B.) et Smith (C.-D.), 1965. - Une épidémie de sporotrichose dans le Vermont associée à de la mousse de sphaigne à l'origine de l'infection. New England J. Med., 272, 1054-1058.

Daugerfield (L.-F.) et Gear (J.), 1941. - Sporotrichosis among miners of the Witwatersrand gold mines. South Afr. Med. J., 15, 128.

DAvis (D.-J.), 1913. - The morphology of Sporothrix schenckii in tissues and in artificial media. J. infect. dis., 12, 453-458.

De Beurmann et Ramond, 1903. - Abcès sous-cutanés multiples d'origine mycosique. Ann. Dermat., 4, 678.

-, et Gougerot, 1906. - Les sporotrichoses hypodermiques, Ann. Derm., 837, 914 et 993.

-, et -, 1907. - Etiologie et pathogénie de la sporotrichose. La tribune médicale, 40, 693696.

-, et -, 1907. - Douzième observation de sporotrichose due au Sporotrichum beurmanni. Chancre sporotrichosique frontal et sporotrichose lymphangitique centripète primitive et localisée. Bull. \& mém. de la Soc. méd. Hôp. Paris, 596.

-, et - 1908. - Découverte du Sporotrichum beurmanni dans la nature. Bull. et mém. de la Soc. méd. Hôp. Paris, 37, 733.

—, et -, 1912. — Les sporotrichoses, 1 vol., 832 p., Alcan éd., Paris.

Du ToIr (C. J.), 1942. - Sporotrichosis on the Witwatersrand, Proceed. Transvaal Mine méd. Off. assoc., 22, 111-127.

Drouhet (E.) et Mariat (F.), 1950. - La pyrimidine, facteur de croissance pour les Sporotrichum. Ann. Inst. Pasteur, 79, 306-313.

-, et -, 1952. - Etude des facteurs déterminant le développement de la phase levure de Sporotrichum schenckii, Ann. Inst. Pasteur, 83, 506-514.

Emmons (C. W.), 1951. - The isolation from soil of fungi which cause disease in man. Trans. N.Y. Acad. Sc., 14, 51-54.

-, 1954. - The significance of saprophytism in the epidemiology of the mycoses. Trans. N.Y. Ac. Sc., Série II, 17 (2), 157-166.

Foerster (H. R.), 1924. - Sporotrichosis. Am. J.M. Sc., 167, 54-75.

—, 1926. - Sporotrichosis, an occupational dermatosis. J.A.M.A., 87, 1605-1609.

GARRET (H. D.) et RoBBins (J. B.), 1960. - An unusual occurence of sporotrichosis. Arch. Derim., 82, 570-571.

Grstineat: (BVN.), Spolyar (L. W.) et Haynes (E.), 1941. - The sporotrichoses. Report of six cases among florists. J.A.M.A., 117, 1074-77. 
GonZalez-Benavides (J.), 1952. - La esporotricosis como enfermedad occupacional de los alfareros. Rev. Hosp. Univ. Monterrey, N.L. Mex., 5, 215.

Gougerot et CARAVEn, 1908. - Sporotrichose spontanée du chien, gommes hypodermiques, péritonite granuleuse et gommes hépatiques, Presse médicale, 43, 337.

GuÉGUEN, 1904. - Les Champignons parasites de l'homme et des animaux. Thèse agrég. Pharmacie, Paris, 250 p.

Hetkoen (L.) et Perkins (C.), 1900. - Refractory subcutaneous abcesses caused by Spororothrix schenckii: new pathogenic fungus. J. Exptl. med., p. 77-89 and J. of the Boston Soc. of Med Sc., p. 169-179.

Howard (D. H.) et ORR (G. F.), 1963. - Comparison of strains of Sporotrichum schenkii isoled from nature. J. Bact., 85, 816-821.

HydE (J. N.) et Davis (D. J.), 1910. - Sporotrichosis in man with consideration of its relation to mycotic lymphangitis in horses. Journ. Cutan. Dis., 28, 321.

L.angeron (M.), 1945. - Précis de Mycologie, 1 vol., 674 p., Masson éd., Paris.

—, et Vanbreuseghem (R.), 1952. - Précis de Mycologie, 1 vol., 703 p., Masson éd., Paris.

Latapi (F.), 1963. - La sporotrichose au Mexique, Laval méd., 34, 732-738.

Lavalle (P.), 1949. - La esporotricosis en Mexico. Trabajo de ingreso a la sociedad Mexicana de Dermatologia.

Lutz (L.), 1948. — Traité de cryptogamie, 1 vol., Masson éd., Paris, 708 p.

—, SPLEndore, 1907. - Sobre una mycose observada em homens e ratos (contribuição para o comhecimento das assim chamadas sporotrichoses). Revista medica de São Paulo.

-, - 1908. - Ueber eine bei Menschen und Ratten beobachtete Mycose. Centralb. f. Bakteriol, 45, 631.

Lwoff (A.) et Monod (J.), 1947. - Essai d'analyse du rôle de l'anhydride carbonique dans la croissance microbienne. Annales Inst. Pasteur, 73, 323-347.

Mackinnon (J. E.), 1948. - Las condiciones meteorologicas causa determinante de la frecuencia de la esporotricosis. An. Insto. Hig. Montevideo, 2, 50-68.

-, 1968. - The effect of temperature of the deep mycoses, In: Systemic mycoses. A Ciba foundation symposium, p. 164-175.

Mariat (F.), 1968. - The epidemiology of the mycosis : some comments in relation to a particular case of sporotrichosis. In: Systemic mycoses. A Ciba foudation symposium, London, Churchill éd., p. 144-159.

Matruchot et RAmond, 1905. - Un type nouveau de champignon pathogène chez l'homme. C.R. Soc. Biol., 59, 379.

Norden (A.), 1951. - Sporotrichosis. Acta pathol. microb. scand., suppl. 89.

Peppler (H. G.) et Twiehaus (M. J.), 1942. - Equine sporotrichosis. Trans. Kans. Acad. Sc., $45,40-46$.

Rogers (A. L.) et BenEKe (E. S.), 1964. - Human pathogenic fungi recovered from Brasilian soil, Mycopathol. Mycol. appl., 22, 15-20.

SALVIN (S. B.), 1947. - Multiple budding in Sporotrichum schenckii Matruchot. J. invest. Dermat., 9, 315-320.

-, 1947. - Cultural studies on the yeast like phase of Histoplasma capsulatum Darling. J. Bact., 54, 655-660 et J. Invest. Dermatol., 9, 315. 
-, 1949. - Cysteine and related compounds in the growth of the yeast like phase of Histoplasma capsulatum. J. Inf. Dis., 84, 275-283.

SARTORY (A.), 1915. - Présence du Sporotrichum beurmanni de Beurmann et Gougerot sur épi de blé. C.R. Soc. Biol., 78, 740.

SCHENCK, 1898. - On refractory subcutaneous abcesses caused by Fungus possibly related to the Sporotricha. John Hopkin's Hospital medical Bulletins, p. 286.

Simson (F. W.), Helm (M. A. F.), Bowen (J. W.) et Brandt (F. A.), 1947. - The pathology of sporotrichosis in man and experimental animals. Proc. Transvaal mine M. offiA., 27, 34-58.

UtTer (M.F.) et Wood (H. G.), 1951. - Mechanisms of fixation of carbon dioxide by heterotrophs and autotrophs. Adv. Enzym., 12, 42-151.

Valle-Meza (A.) et Barba-Rubio (M.), 1961. - Esporotricosis facial en un lactante. Mem. 1. Congr. Mex. Derm. 